\title{
Mutual or Unrequited Love: Identifying Stable Clusters in Social Networks with Uni- and Bi-directional Links
}

\author{
Yanhua Li, Zhi-Li Zhang and Jie Bao \\ Department of Computer Science \& Engineering, University of Minnesota, Twin Cities \\ Email: \{yanhua,zhzhang,baojie\}@cs.umn.edu
}

\begin{abstract}
Many social networks, e.g., Slashdot and Twitter, can be represented as directed graphs (digraphs) with two types of links between entities: mutual (bi-directional) and one-way (uni-directional) connections. Social science theories reveal that mutual connections are more stable than one-way connections, and one-way connections exhibit various tendencies to become mutual connections. It is therefore important to take such tendencies into account when performing clustering of social networks with both mutual and one-way connections.

In this paper, we utilize the dyadic methods to analyze social networks, and develop a generalized mutuality tendency theory to capture the tendencies of those node pairs which tend to establish mutual connections more frequently than those occur by chance. Using these results, we develop a mutuality-tendencyaware spectral clustering algorithm to identify more stable clusters by maximizing the within-cluster mutuality tendency and minimizing the cross-cluster mutuality tendency. Extensive simulation results on synthetic datasets as well as real online social network datasets such as Slashdot, demonstrate that our proposed mutuality-tendency-aware spectral clustering algorithm extracts more stable social community structures than traditional spectral clustering methods.
\end{abstract}

\section{INTRODUCTION}

Graph models are widely utilized to represent relations among entities in social networks. Especially, many online social networks, e.g., Slashdot and Twitter, where the users' social relationships are represented as directed edges in directed graphs (or in short, digraphs). Entity connections in a digraph can be categorized into two types, namely, bidirectional links (mutual connections) and uni-directional links (one-way connections). Social theories [28] and online social network analysis [2], [7], [28] have revealed that various types of connections exhibit different stabilities, where mutual connections are more stable than one-way connections. In other words, mutual connections are the source of social cohesion [3], [4] that, if two individuals mutually attend to one another, then the bond is reinforced in each direction.

Studying the social network structure and properties of social ties have been an active area of research. Clustering and identifying social structures in social networks is an especially important problem [9], [17], [24] that has wide applications, for instance, community detection and friend recommendation in social networks. Existing clustering methods [21], [29] are originally developed for undirected graphs, based on the

The work is supported in part by the NSF grants CNS-0905037, CNS1017647 and the DTRA Grant HDTRA1-09-1-0050. classical spectral clustering theory. Several recent studies (see, e.g., [11], [21], [27], [29]) extend the spectral clustering method to digraphs, by first converting the underlying digraphs to undirected graphs via some form of symmetrization, and then apply spectral clustering to the resulting symmetrized (undirected) graphs. However, all these methods have two common drawbacks, which prevent them from obtaining stable clusters with more mutual connections. First, these methods do not explicitly distinguish between mutual and one-way connections commonly occurring in many social networks, treating them essentially as the same and therefore ignoring the different social relations and interpretations these two types of connections represent (see Section $\Pi$ for more in-depth discussion). Second, by simply minimizing the total cross-

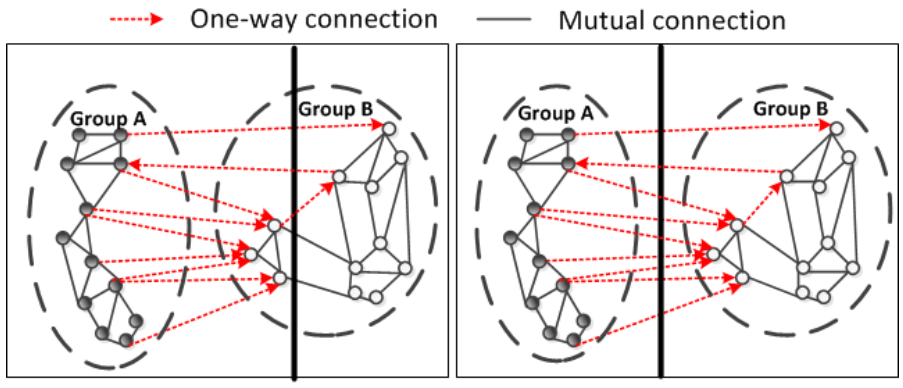

(a) Traditional Spectral Clustering

(b) Tendency Aware Spectral Clustering

Fig. 1. An example network

cluster links (that are symmetrized in some fashion), these methods do not explicitly account for the potential tendencies of node pairs to become mutually connected. As a simple example, Fig. 1 shows two groups of people in a network, where people in the same group tend to have more mutual (stable) connections, and people across two groups have more one-way (unstable) connections. When using the traditional spectral clustering method, as shown in Fig. 11a), group B will be partitioned into two clusters, due to its strict rule of minimizing the total number of across cluster edges. On the other hand, the correct partition should be done as shown in Fig. 1 b), where the majority of mutual (stable) connections are placed within clusters, and one-way (unstable) connections are placed across clusters.

In this paper, we propose and develop a stable social cluster detection algorithm that takes into account the tendencies of node pairs whether to form mutual (thus stable) connections 
or not, which can result in more stable cluster structures. To tackle this clustering problem, we need to answer the following questions: 1) how to track and evaluate the tendencies of node pairs to become mutual (stable) relations? and 2) how to cluster the entities in social networks by accounting for their mutuality tendencies so as to extract more stable clustering structures?

To address these questions, we utilize dyadic methods to analyze social networks, and develop a generalized mutuality tendency theory which better captures the tendencies of node pairs that tend to establish mutual connections more frequently than those occur by chance. Using these results, we develop a mutuality-tendency-aware spectral clustering algorithm to detect more stable clusters by maximizing the within-cluster mutuality tendency and minimizing the cross-cluster mutuality tendency. Our contributions are summarized as follows.

- Motivated by the social science mutuality tendency theory, we establish a new cluster-based mutuality tendency theory which yields a symmetrized mutuality tendency for each node pair, and provides a measure of strength of social ties among nodes in a cluster.

- Based on our theory, we develop a mutuality-tendencyaware spectral clustering algorithm that can partition the social graphs into stable clusters, by maximizing the within-cluster mutuality tendencies and minimizing the across cluster mutuality tendencies.

- The experimental results - based on both social network structures of synthetical and real social network datasets - confirm that our clustering algorithm is able to generate more stable clusters than the traditional spectral clustering algorithms.

To our best knowledge, this is the first work studying the impact of tendencies of node pairs to become mutual connections on the stability of cluster structure of social networks. The remainder of the paper is organized as follows. In section briefly discuss the existing dyadic analysis methods, the traditional spectral clustering algorithms and other related work. In section П we introduce a cluster-base mutuality tendency theory, and based on this theory, we develop a mutualitytendency-aware spectral clustering algorithm in section IV In section $\mathrm{V}$, we evaluate the performances of our method using synthetic and real social network (e.g., Slashdot) datasets. We conclude the paper in section VI.

\section{Preliminaries, Related Work And Problem DEFINITION}

In this section, we first introduce the existing dyadic analysis methods in the social theory literature for analyzing and characterizing social network mutual connections and one-way connections. We then present the classic spectral clustering theory which was developed for undirected graphs, and briefly survey some related works which apply this theory to digraphs through symmetrization. We argue that these existing methods for clustering digraphs via symmetrization are inadequate in solving social network clustering problems, as they ignore different social ties (and mutuality tendencies) represented by mutual and one-way connections in social networks. We end the section with the problem definition, namely, how to identify stable clusters in social networks by taking into account mutuality tendencies of mutual and one-way connections.

\section{A. Dyadic Analysis and Mutuality Tendency}

Given a social network with both uni- and bi-directional links, such a network can be represented as a (simple) digraph $G=(V, E)$ with $|V|=n$ nodes. If the links also have weights (say, representing the strength of connections or social ties), such a network can be more generally represented as a weighted digraph, $G=(V, E, A)$ where $A_{i j}$ represents the strength of connection or "affinity" from node $i$ to node $j$. When $A$ is a $0-1$ matrix, $G$ reduces to a simple digraph, and $A$ is the standard adjacency matrix of the digraph, where $A_{i j}=1$ if the directed edge $i \rightarrow j$ is present, and $A_{i j}=0$ otherwise. In this paper for simplicity we focus primarily on simple (unweighted) digraphs with no selfloops, namely, social networks with unweighted directional links. Most online social networks are of unweighted variety.

Social scientists commonly view the social network $G$ as a collection of dyads [28], where a dyad is an unordered pair of nodes and directed edges between two nodes in the pair. Denote a dyad as $D y_{i j}=\left(A_{i j}, A_{j i}\right)$, for $i<j$. Since dyad is an unordered notion, we have in total $N_{d}=n(n-1) / 2$ dyads in $G$. Hence, there are only three possible isomorphism dyads. The first type of dyads is mutual relationship, where both directional edges $i \rightarrow j$ and $j \rightarrow i$ are present. The second type of dyads is one-way relationship, where either $i \rightarrow j$ or $j \rightarrow i$ is present, but not both. The last type of dyads is null relationship, where no edges show up between $i$ and $j$.

Interpretations of dyads. Social scientists have observed that mutual social relations and one-way relations in social networks typically exhibit different stabilities, namely, mutual relations are more stable than one-way relations [28]. Hence in the social science literature, one prevalent interpretation of dyadic relations in social networks are the following: mutual dyads are considered as stable connections between two nodes and null relation dyads represent no relations; the one-way dyads [1], [6], [16], [18], [20] are viewed as an intermediate state of relations, which are in transition to more stable equilibrium states of reciprocity (mutual or no relation). Several recent empirical studies [7], [10] of online social networks have further revealed and confirmed that mutual social relations are more stable relations than oneway connections.

Computing dyad census. Given a (simple) digraph $G=$ $(V, E)$, with $n=|V|$ nodes. Let $m, b$, and $u$ denote the number of mutual, one-way, and null dyads in the network. Clearly, $m+b+u=n(n-1) / 2$. The triple $\langle m, b, u\rangle$ is referred to as the dyad census, since it is derived from an examination of all (possible) dyads in the network. The dyad census triple can be computed in terms of the adjacency matrix $A$ of $G$ as 
follows (in both scalar and matrix forms):

$$
\begin{aligned}
& m=\sum_{i<j} A_{i j} A_{j i}=\frac{1}{2} \operatorname{tr}(A A), \\
& b=|E|-2 m=\operatorname{tr}\left(A A^{T}\right)-\operatorname{tr}(A A), \\
& u=N_{d}-b-m=N_{d}-\operatorname{tr}\left(A A^{T}\right)+\operatorname{tr}(A A) .
\end{aligned}
$$

Measuring mutuality tendency. The notion of mutuality tendency has been introduced in the social science literature (see, e.g., [8], [28]) to measure the tendency for a node pair to establish mutual connections. For any dyad between $i$ and $j$ in a digraph $G$, if $i$ places a link to $j, \rho_{i j}$ represents the tendency that $j$ will reciprocate to $i$ more frequently than would occur by chance.

Let $\mathbf{X}_{i j}$ denote the random variable that represents whether or not node $i$ places a directed edge to node $j$. There are only two possible events (i.e., $\mathbf{X}_{i j}$ takes two possible values): $\mathbf{X}_{i j}=$ 1 , representing the edge is present; or $\mathbf{X}_{i j}=0$, the edge is not present. Let $X_{i j}$ (resp. $\bar{X}_{i j}$ ) denote the event $\left\{\mathbf{X}_{i j}=1\right\}$ (resp. $\left.\left\{\mathbf{X}_{i j}=0\right\}\right)$. Then the probability of the event $X_{i j}$ occurring is $P\left(X_{i j}\right)$. The probability that $i$ places a directed edge to $j$ and $j$ reciprocates back (i.e., node $i$ and node $j$ are mutually connected) is thus given by

$$
P\left(X_{i j}, X_{j i}\right)=P\left(X_{i j}\right) P\left(X_{j i} \mid X_{i j}\right),
$$

Wofle [28] introduces the following measure of mutuality tendency in terms of the conditional probability $P\left(X_{j i} \mid X_{i j}\right)$ as follows:

$$
\begin{aligned}
& P\left(X_{j i} \mid X_{i j}\right)=P\left(X_{j i}\right)+\rho_{i j} P\left(\bar{X}_{j i}\right), \\
& \rho_{i j}=\frac{P\left(X_{i j}, X_{j i}\right)-P\left(X_{i j}\right) P\left(X_{j i}\right)}{P\left(X_{i j}\right) P\left(\bar{X}_{j i}\right)},
\end{aligned}
$$

where $-\infty<\rho_{i j} \leq 1$ ensures $0 \leq P\left(X_{j i}\right)+\rho P\left(\bar{X}_{j i}\right) \leq 1$ to hold. Like many indices used in statistics, $\rho_{i j}$ is dimensionless and easy to interpret, since it uses 0 and 1 as benchmarks. If $\rho=1$, the mutuality tendency is maximum, meaning that given that node $i$ places a link to node $j$, node $j$ will for sure reciprocate. If $\rho_{i j}=0$ (i.e., $P\left(X_{j i} \mid X_{i j}\right)=P\left(X_{j i}\right)$ ), then node $j$ reciprocates and places a link to node $i$ purely by chance, namely, it is independent of the event that node $i$ places a link to node $j$. Hence when $0<\rho_{i j} \leq 1$, it suggests more than a chance tendency for node $j$ to reciprocate back. Furthermore, if $\rho_{i j}<0$ (i.e., $P\left(X_{j i} \mid X_{i j}\right)<P\left(X_{j i}\right)$ ), there is less than chance tendency for node $j$ to reciprocate; in other words, it suggests a tendency away from mutual dyads, toward one-way and null dyads. Hence, $-\infty<\rho_{i j} \leq 1$ provides a measure of the strength of tendency for reciprocation.

From eq. (2), the joint distribution $P\left(X_{i j}, X_{j i}\right)$ in eq. (2) can be measured by the observed graph, namely, either $P\left(X_{i j}, X_{j i}\right)=P^{(\omega)}\left(X_{i j}, X_{j i}\right)=1$, when $i$ and $j$ have mutual connection, or $P\left(X_{i j}, X_{j i}\right)=P^{(\omega)}\left(X_{i j}, X_{j i}\right)=0$, otherwise, where the superscript $\omega$ indicates that the probability is obtained from the observed graph. On the other hand, the distribution for each individual edge is measured by $P\left(X_{i j}\right)=P^{(\mu)}\left(X_{i j}\right)=\frac{d_{i}}{|V|-1}$, where $d_{i}$ is the out-going degree of node $i . P^{(\mu)}\left(X_{i j}\right)$ represents the probability of edge $i \rightarrow j$ being generated under a random graph model, denoted by the superscript $\mu$, with edges randomly generated while preserving the out-degrees. Hence, the tendency $\rho_{i j}$ is obtained by implicitly comparing the observed graph with a reference random digraph model.

Limitations of Wolfe's mutuality tendency measure for stable social structure clustering. Although the node pair in a dyad is unordered (i.e., the two nodes are treated "symmetrically" in terms of dyadic relations), Wolfe's measure of mutual tendency is in fact asymmetric. This can be easily seen through the following derivation. By definition,

$$
\begin{aligned}
& P\left(X_{j i} \mid X_{i j}\right)=P\left(X_{j i}\right)+\rho_{j i} P\left(\bar{X}_{j i}\right), \\
& P\left(X_{i j} \mid X_{j i}\right)=P\left(X_{i j}\right)+\rho_{i j} P\left(\bar{X}_{i j}\right) .
\end{aligned}
$$

Multiplying the above two equations with $P\left(X_{i j}\right)$ and $P\left(X_{j i}\right)$ respectively and from eq.(1), we have

$$
\frac{\rho_{j i}}{\rho_{i j}}=\frac{P\left(X_{j i}\right) P\left(\bar{X}_{i j}\right)}{P\left(X_{i j}\right) P\left(\bar{X}_{j i}\right)}=\frac{P\left(X_{j i}\right)-P\left(X_{i j}\right) P\left(X_{j i}\right)}{P\left(X_{i j}\right)-P\left(X_{i j}\right) P\left(X_{j i}\right)}
$$

We see that $\rho_{i j}=\rho_{j i}$ if and only if $P\left(X_{i j}\right)=P\left(X_{j i}\right)$ holds. Hence, given an arbitrary dyad in a social network Wolfe's measure of mutuality tendency of the node pair is asymmetric - in a sense that it is a node-specific measure of mutuality tendency. It does not provide a measure of mutuality tendency of the (unordered) node pair viewed together. While such asymmetric (node-specific) measure of mutuality tendency can be useful in some social network analysis, as will be clear later, such an asymmetric measure poses difficulty in identifying and extracting stable cluster structures in social networks. For instance, given a partition $V=(S, \bar{S})$ of a digraph, generalizing Wolfe's measure to clusters, the mutuality tendencies across the two clusters, denoted by $\rho(S, \bar{S})=\sum_{i \in S, j \in \bar{S}} \rho_{i j}$ and $\rho(\bar{S}, S)=\sum_{i \in \bar{S}, j \in S} \rho_{i j}$, are generally not symmetric, namely, $\rho(S, \bar{S}) \neq \rho(\bar{S}, S)$. In Section III we will introduce a new measure of mutuality tendency that is symmetric and captures the tendency of a node pair in a dyadic relation to establish mutual connection. This measure of mutuality tendency can be applied to clusters and a whole network in a straightforward fashion, and leads us to develop a mutuality-tendency-aware spectral clustering algorithm.

\section{B. Spectral Clustering Theory and Extensions to Digraphs via Symmetrization}

Spectral clustering methods (see, e.g., [15], [22], [26], [27], [29]) are originally developed for clustering data with symmetric relations, namely, data that can be represented as undirected graphs, where each relation (edge) between two entities, $A_{i j}=A_{j i}$, represents their similarity. The goal is to partition the graph such that entities within each cluster are more similar to each other than those across clusters. This is done by minimizing the total weight of cross-cluster edges (possibly weighted by the total weight of edges within clusters). In the following we present the basics of spectral clustering theory (see [25] for more details). 
Given the (non-negative) similarity matrix $A$, the cut function is defined to quantatively measure the quality of a partition $V=\left(S_{1}, \cdots, S_{K}\right)$, and is defined as follows:

$$
\begin{aligned}
\operatorname{Cut}\left(S_{l}, \bar{S}_{l}\right) & :=\sum_{i \in S_{l}, j \in \bar{S}_{l}} A_{i j}, \\
\operatorname{Cut}\left(S_{1}, \cdots, S_{K}\right) & :=\sum_{i=1}^{K} \operatorname{Cut}\left(S_{i}, \bar{S}_{i}\right) .
\end{aligned}
$$

To account for cluster sizes - especially to obtain relatively balanced clusters (in terms of sizes), the ratio cut function RCut [5] and the normalized cut function NCut [22] have also been defined:

$$
\begin{aligned}
\operatorname{RCut}\left(S_{1}, \cdots, S_{K}\right) & :=\sum_{i=1}^{K} \frac{\operatorname{Cut}\left(S_{i}, \bar{S}_{i}\right)}{\left|S_{i}\right|}, \\
\operatorname{NCut}\left(S_{1}, \cdots, S_{K}\right) & :=\sum_{i=1}^{K} \frac{\operatorname{Cut}\left(S_{i}, \bar{S}_{i}\right)}{\operatorname{Vol}\left(S_{i}\right)},
\end{aligned}
$$

where $\operatorname{vol}\left(S_{i}\right)=\sum_{j \in S_{i}} d_{j}$ is the volume of the cluster $S_{i}$.

In the following (and the remainder of the paper), we will use the ratio cut function as the objective function. All the results also hold true for the normalized cut. Using the ratio cut, the clustering problem formulated as a graph mincut optimization problem can be rewritten in the following form:

$$
\min _{S_{1}, \cdots, S_{k}} \operatorname{RCut}\left(S_{1}, \cdots, S_{K}\right),
$$

The (unnormalized) Laplacian matrix $L=D-A$ is used to solve the above mincut problem, where $D=\operatorname{diag}\left[d_{i}\right]$ with $d_{i}=\sum_{j} A_{i j}$ is the diagonal degree matrix. Given a (nonnegative) symmetric $A, L$ is symmetric and positive semi-definite. If we take $K$ eigenvectors corresponding to the smallest eigenvalues of $L$, the optimal solution to the problem eq.(3), namely, the optimal partition into $K$ clusters, can be well approximated by applying the K-means algorithm to clustering the data points projected to the subspace formed by these $K$ eigenvectors [25]. Moreover, [13] provides a systematic study on comparing a wide range of undirected graph based clustering algorithms using real large datasets, which gives a nice guideline of how to select clustering algorithms based on the underlying networks and the targeting objectives.

Extensions to digraphs via symmetrization. When relations between entities are asymmetric, or the underlying graph is directed, spectral clustering cannot be directly applied, as the notion of (semi-)definiteness is only defined for symmetric matrices. Several recent studies (see, e.g., [11], [21], [27], [29]) all attempt to circumvent this difficulty by first converting the underlying digraphs to undirected graphs via some form of symmetrization, and then apply spectral clustering to the resulting symmetrized (undirected) graphs. For example, the authors in [21] discuss several symmetrization methods, including the symmetrized adjacency matrix $\bar{A}=\left(A+A^{T}\right) / 2$, the bibliographic coupling matrix $A A^{T}$ and the co-citation strength matrix $A^{T} A$, and so forth. Symmetrization can also be done through a random walk on the underlying graph, where $P=D^{-1} A$ is the probability transition matrix and $D=\operatorname{diag}\left[d_{i}^{\text {out }}\right]$ is a diagonal matrix of node out-degrees. For example, taking the objective function as the random walk flow circulation matrix $F_{\pi}=\Pi P$, where $\Pi$ is the diagonal stationary distribution matrix, we have the symmetrized Laplacian of the circulation matrix as

$$
\overline{\mathcal{L}}=\frac{\tilde{\mathcal{L}}+\tilde{\mathcal{L}}^{T}}{2}=I-\frac{\Pi^{\frac{1}{2}} P \Pi^{-\frac{1}{2}}+\Pi^{-\frac{1}{2}} P^{T} \Pi^{\frac{1}{2}}}{2} .
$$

where $\tilde{\mathcal{L}}$ is the (asymmetric) digraph Laplacian matrix [14]. Then the classical spectral clustering algorithm can then be applied using $\overline{\mathcal{L}}$ which is symmetric and semi-definite. Zhou and et al [27], [29] use this type of symmetrization to perform clustering on digraphs. Moreover, Leicht and Newman [11] propose the digraph modularity matrix $Q=\left[Q_{i j}\right]$, which captures the difference between the observed digraph and the hypothetical random graph with edges randomly generated by preserving the in- and out-degrees of nodes, namely, $Q_{i j}=A_{i j}-d_{i}^{o u t} d_{j}^{i n} / m$. Then, if the sum of edge modularities in a cluster $S$ is large, nodes in $S$ are well connected, since the edges in $S$ tend to appear with higher probabilities than occur by chance. However, $Q$ by definition is asymmetric, where [11] uses the symmetrized $\bar{Q}=\left(Q+Q^{T}\right) / 2$ as objective to perform spectral clustering method. Essentially, the edge modularity captures how an individual edge appears more frequently than that happens by chance, thus the modularity based clustering method tends to group those nodes with more connections than expected together, which like all other clustering methods presented above completely ignores the distinction between mutual and one-way connections.

Problem definition: Clustering and identifying stable clusters in social networks with mutual and one-way connections. As discussed earlier, one-way and mutual dyadic connections in social networks often represent different states or types of social ties and exhibit various stabilities over time. Hence when performing clustering to extract community structures in social networks, one-way and mutual connections should be distinguished and treated differently. Existing digraph clustering methods via symmetrization, e.g., those mentioned above, on the other hand, ignore these different types of connections and treat them as the same: the process of symmetrization essentially weighs one-way connections as a fraction of mutual connections, and then attempt to minimize the total weight of the (symmetrized) cross-cluster links. Moreover, different from Leicht and Newman's [11] reference random graph model, as presented in earlier section, the mutuality tendency compares the observed the digraph with a random graph model where edges are randomly generated by preserving only the out-degrees, which better reflects the underlying model of how social network users establish social ties.

In this paper we want to solve the following clustering problem in social networks with bi- and uni-directional links: Given a directed (social) graph where mutual connections represent more stable relations and one-way connections rep- 
resent intermediate transferring states, how can we account for mutual tendencies of dyadic relations and cluster the entities in such a way that nodes within each cluster have maximized mutuality tendencies to establish mutual connections, while across clusters, nodes have minimized tendencies to establish mutual connections? The clusters (representing social structures or communities) identified and extracted thereof will hence likely be more stable.

\section{Cluster-Based Mutuality Tendency Theory}

Inspired by Wolfe's study in [28], we propose a new measure of mutuality tendency for dyads that can be generalized to groups of nodes (clusters), and develop a mutuality tendency theory for characterizing the strength of social ties within a cluster (network structure) as well as across clusters in an asymmetric social graph. This theory lays the theoretical foundation for the network structure classification and community detection algorithms we will develop in section IV

\section{A. Cluster based mutuality tendency}

Let $\mathbf{X}_{i j}$ denote the random variable that represents whether or not node $i$ places a directed edge to node $j$. There are only two possible events (i.e., $\mathbf{X}_{i j}$ takes two possible values): $\mathbf{X}_{i j}=1$, representing the edge is present; or $\mathbf{X}_{i j}=0$, the edge is not present. Let $X_{i j}$ (resp. $\bar{X}_{i j}$ ) denote the event $\left\{\mathbf{X}_{i j}=1\right\}$ (resp. $\left\{\mathbf{X}_{i j}=0\right\}$ ). Given an observed (asymmetric) social graph $G$, to capture the mutuality tendency of dyads in this graph, we compare it with a hypothetical, random (social) graph, denoted as $G^{(\mu)}$, where links (dyadic relations) are generated randomly (i.e., by chance) in such a manner that the (out-)degree $d_{i}$ of each node $i$ in $G^{(\mu)}$ is the same as that in the observed social graph $G$. Under this random social graph model, the probability of the event $X_{i j}$ occurring is $P^{(\mu)}\left(X_{i j}\right)=\frac{d_{i}}{|V|-1}$; namely, $i$ places a (directed) link to node $j$ randomly or by chance (the superscript $\mu$ indicates the probability distribution of link generations under the random social graph model). The probability that $i$ places a directed edge to $j$ and $j$ reciprocates back (i.e., node $i$ and node $j$ are mutually connected) is thus given by $P^{(\mu)}\left(X_{i j}, X_{j i}\right)=$ $P^{(\mu)}\left(X_{i j}\right) P^{(\mu)}\left(X_{j i} \mid X_{i j}\right)=P^{(\mu)}\left(X_{i j}\right) P^{(\mu)}\left(X_{j i}\right)$, since $\mathbf{X}_{i j}$ and $\mathbf{X}_{j i}$ are independent under the random social graph model. On the observed social graph, denote $P^{(\omega)}\left(X_{i j}, X_{j i}\right)$ to represent the event whether there is a mutual connection (symmetric link) between node $i$ and node $j$, i.e., $P^{(\omega)}\left(X_{i j}, X_{j i}\right)=1$, if the dyad $D y_{i j}$ is a mutual dyad in the observed social graph, and $P^{(\omega)}\left(X_{i j}, X_{j i}\right)=0$, otherwise. We define the mutuality tendency of dyad $D y_{i j}$ as follows:

$$
\begin{aligned}
\theta_{i j}: & =P^{(\omega)}\left(X_{i j}, X_{j i}\right)-P^{(\mu)}\left(X_{i j}, X_{j i}\right) \\
& =P^{(\omega)}\left(X_{i j}, X_{j i}\right)-P^{(\mu)}\left(X_{i j}\right) P^{(\mu)}\left(X_{j i}\right),
\end{aligned}
$$

which captures how the node pair $i$ and $j$ establish a mutual dyad more frequently than would occur by chance.

This definition of mutuality tendency is a symmetric measure for dyad $D y_{i j}$, i.e., $\theta_{i j}=\theta_{j i}$. In addition, it is shown that $\theta_{i j} \in[-1,1]$. We remark that $\theta_{i j}=0$ indicates that if node $i$ places a directed link to node $j$, the tendency that node $j$ will reciprocate back to node $i$ is no more likely than would occur by chance; the same holds true if node $j$ places a directed link to node $i$ instead. On the other hand, $\theta_{i j}>0$ indicates that if node $i$ (resp. node $j$ ) places a directed link to node $j$ (resp. node $i$ ), node $j$ (resp. node $i$ ) will more likely than by chance to reciprocate. In particular, with $\theta_{i j}=1$, node $j$ (resp. node $i)$ will almost surely reciprocate. In contrast, $\theta_{i j}<0$ indicates that if node $i$ (resp. node $j$ ) places a directed link to node $j$ (resp. node $i$ ), node $j$ (resp. node $i$ ) will tend not to reciprocate back to node $i$ (resp. node $j$ ). In particular, with $\theta_{i j}=-1$, node $j$ (resp. node $i$ ) will almost surely not reciprocate back. Hence $\theta_{i j}$ provides a measure of strength of social ties between node $i$ and $j: \theta_{i j}>0$ suggests that the dyadic relation between node $i$ and $j$ is stronger, having a higher tendency (than by chance) to become mutual; whereas $\theta_{i j}<0$ suggests that node $i$ and $j$ have weaker social ties, and their dyadic relation is likely to remain asymmetric or eventually disappear.

Mutuality tendency of clusters. The mutuality tendency measure for dyads defined in eq. (4) can be easily generalized for an arbitrary cluster (a subgraph) in an observed social graph, $S \subseteq G$. We define the mutuality tendency of a cluster $S, \Theta_{S}$, as follows:

$$
\begin{aligned}
& \Theta_{S}:=\sum_{i \sim j ; i, j \in S} P^{(\omega)}\left(X_{i j}, X_{j i}\right)-\sum_{i \sim j ; i, j \in S} P^{(\mu)}\left(X_{i j}, X_{j i}\right) \\
& =\sum_{i \sim j ; i, j \in S} P^{(\omega)}\left(X_{i j}, X_{j i}\right)-\sum_{i \sim j ; i, j \in S} P^{(\mu)}\left(X_{i j}\right) P^{(\mu)}\left(X_{j i}\right),
\end{aligned}
$$

where the subscript $i \sim j: i, j \in S$ means that the summation accounts for all (unordered) dyads, and $i, j$ are both in $S$. Denote the second term in eq. (5) as $m_{S}^{(\mu)}$, and the (out-degree) volume of the cluster $S$ as $d_{S}:=\sum_{i \in S} d_{i}$. As $P^{(\mu)}\left(X_{i j}\right)=$ $d_{i} /(|V|-1)$ and $P^{(\mu)}\left(X_{j i}\right)=d_{j} /(|V|-1)$,

$$
m_{S}^{(\mu)}=\sum_{i \sim j ; i, j \in S} \frac{d_{i} d_{j}}{(|V|-1)^{2}}=\frac{d_{S}^{2}-\sum_{i \in S} d_{i}^{2}}{2(|V|-1)^{2}}
$$

which represents the expected number of mutual connections among nodes in $S$ under the random social graph model. Given the cluster $S$ in the observed social graph $G$, define $m_{S}^{(\omega)}:=\sum_{i \sim j ; i, j \in S} P^{(\omega)}\left(X_{i j}, X_{j i}\right)$, namely, $m_{S}^{(\omega)}$ represents the number of (observed) mutual connections among nodes in the cluster $S$ in the observed social graph $G$. The mutual tendency of cluster $S$ defined in eq. (5) is therefore exactly $\Theta_{S}=m_{S}^{(\omega)}-m_{S}^{(\mu)}$.

Hence $\Theta_{S}$ provides a measure of strength of (likely mutual) social ties among nodes in a cluster: $\Theta_{S}>0$ suggests that there are more mutual connections among nodes in $S$ than would occur by chance; whereas $\Theta_{S}<0$ suggests that there are fewer mutual connections among nodes in $S$ than would occur by chance. Using $\Theta_{S}$, we can therefore quantify and detect clusters of nodes (network structures or communities) that have strong social ties.

In particular, when $S=G, \Theta_{G}$ characterizes the mutuality 
tendency for the entire digraph $G$, i.e.,

$$
\Theta_{G}=m_{G}^{(\omega)}-m_{G}^{(\mu)}=\sum_{i \sim j} \theta_{i j},
$$

where $m_{G}^{(\omega)}:=\sum_{i \sim j} P^{(\omega)}\left(X_{i j}, X_{j i}\right)$ represents the number of (observed) mutual dyads among nodes in the observed social graph $G$, and

$$
m_{G}^{(\mu)}=\sum_{i \sim j} \frac{d_{i} d_{j}}{(|V|-1)^{2}}=\frac{d^{2}-\sum_{i \in V} d_{i}^{2}}{2(|V|-1)^{2}},
$$

represents the expected number of mutual dyads among nodes in $G$ under the random social graph model. Likewise, given a bipartition $(S, \bar{S})$ of $G$, we define the cross-cluster mutuality tendency as

$$
\Theta_{\partial S}:=\sum_{i \in S \sim j \in \bar{S}}\left(P^{(\omega)}\left(X_{i j} X_{j i}\right)-P^{(\mu)}\left(X_{i j}\right) P^{(\mu)}\left(X_{j i}\right)\right)
$$

Denote the second quantity in eq.9) as $m_{S}^{(\mu)}$,

$$
m_{\partial S}^{(\mu)}=\sum_{i \in S \sim j \in \bar{S}} \frac{d_{i} d_{j}}{(|V|-1)^{2}}=\frac{d_{S} d_{\bar{S}}}{(|V|-1)^{2}}
$$

which represents the expected number of mutual connections among nodes across $S$ and $\bar{S}$ under the random social graph model. Define $m_{\partial S}^{(\omega)}:=\sum_{i \in S \sim j \in \bar{S}} P^{(\omega)}\left(X_{i j}, X_{j i}\right)$ representing the number of (observed) mutual connections among nodes across clusters $S$ and $\bar{S}$ in the observed social graph $G$. The mutuality tendency across cluster $S$ and $\bar{S}$ defined in eq.91 is therefore exactly $\Theta_{\partial S}=m_{\partial S}^{(\omega)}-m_{\partial S}^{(\mu)}$.

The mutuality tendency theory outlined above accounts for different interpretations and roles mutual and one-way connections represent and play in asymmetric social graphs, with the emphasis in particular on the importance of mutual connections in forming and developing stable social structures/communities with strong social ties. In the next section, we will show how we can apply this mutuality tendency theory for detecting and clustering stable network structures and communities in asymmetric social graphs.

\section{Mutuality-Tendency-AWARE SPECTRAL CLUSTERING ALGORITHM}

In this section, we first consider the simpler case of mutuality-tendency-aware clustering problem with $K=2$ and establish the basic theory and algorithm. We then extend it to the general case with $K>2$.

\section{A. Mutuality-tendency-aware spectral clustering: $K=2$}

Without loss of generality, we consider only simple (unweighted) digraphs $G=(V, E)$ (i.e., the adjacency matrix $A$ is a $0-1$ matrix). Define the mutual connection matrix $M:=\min \left(A, A^{T}\right)$, which expresses all the mutual connections with unit weight 1 . In other words, if node $i$ and node $j$ are mutually connected (with bidirectional links), $M_{i j}=M_{j i}=1$, otherwise, $M_{i j}=M_{j i}=0$. Hence, we have $M_{i j}=P^{(\omega)}\left(X_{i j}, X_{j i}\right)$, representing the event whether there is a mutual connection (symmetric link) between node $i$ and node $j$, i.e., in the dyad $D y_{i j}$ in the observed social graph. In addition, let $\delta_{i j}$ be the Kronecker delta symbol, i.e., $\delta_{i j}=1$ if $i=j$, and $\delta_{i j}=0$ otherwise. Then, we define matrix

$$
\bar{M}=\frac{d d^{T}-\operatorname{diag}\left[d^{2}\right]}{(|V|-1)^{2}}
$$

with $d$ as the out-going degree vector, where each entry

$$
\bar{M}_{i j}=\frac{d_{i} d_{j}-\delta_{i j} d_{i}^{2}}{(|V|-1)^{2}}= \begin{cases}\frac{d_{i} d_{j}}{(|V|-1)^{2}} & \text { if } i \neq j \\ 0 & \text { if } i=j\end{cases}
$$

represents the probability that two nodes $i$ and $j$ independently place two unidirectional links to each other to form a mutual dyad. Hence, $\bar{M}_{i j}=P^{(\mu)}\left(X_{i j}\right) P^{(\mu)}\left(X_{j i}\right)$ represents the probability of node pair $i$ and $j$ to establish a mutual connection under random graph model with edges randomly generated by preserving the node out-degrees. We denote $T=M-\bar{M}$ as the mutuality tendency matrix, with each entry

$$
T_{i j}=P^{(\omega)}\left(X_{i j}, X_{j i}\right)-P^{(\mu)}\left(X_{i j}\right) P^{(\mu)}\left(X_{j i}\right)=\theta_{i j}
$$

as the individual dyad mutuality tendency.

Mutuality Tendency Lapacian. $T$ is symmetric and those entries associated with non-mutual dyads are negative, representing less mutuality tendencies to establish mutual connections than those occur by chance. Define the mutuality tendency Laplacian matrix as

$$
L_{T}=D_{T}-T
$$

where $D_{T}=\operatorname{diag}\left[d_{T}(i)\right]$ is the diagonal degree matrix of $T$, with $d_{T}(i)=\sum_{j} T_{i j}$. We have the following theorem presenting several properties of $L_{T}$.

Theorem 1. The mutuality tendency Laplacian matrix $L_{T}$ as defined in eq.(13) has the following properties

- Given a column vector $x \in \mathbb{R}^{|V|}$, the bilinear form $x^{T} L_{T} x$ satisfies

$$
x^{T} L_{T} x=\sum_{i \sim j} T_{i j}\left(x_{i}-x_{j}\right)^{2} .
$$

- $L_{T}$ is symmetric and in general indefinite. In addition, $L_{T}$ has one eigenvalue equal to 0 , with corresponding eigenvector as $\mathbf{1}=[1, \cdots, 1]^{T}$.

Proof : (1) By expanding the bilinear form $x^{T} L_{T} x$,

$$
x^{T} L_{T} x=\sum_{i, j \in V} T_{i j}\left(x_{i}^{2}-x_{i} x_{j}\right)=\sum_{i \sim j} T_{i j}\left(x_{i}-x_{j}\right)^{2}
$$

(2) The symmetry of both $M$ and $\bar{M}$ in eq.12 insures the symmetry of $L_{T}$, thus $L_{T}$ has all real eigenvalues. However, $L_{T}$ is in general indefinite, because $T_{i j}$ in eq. (14) could be either positive or negative. On the other hand, since $\mathbf{1}^{T} L_{T}=$ $\mathbf{0}^{T}$ and $L_{T} \mathbf{1}^{T}=\mathbf{0}$ hold true, $L_{T}$ has an eigenvalue equal to 0 with corresponding eigenvectors as identity vector $\mathbf{1}=$ $[1, \cdots, 1]^{T}$. 
Mutuality tendency ratio cut function. For a digraph $G=$ $(V, E)$, and a partition $V=(S, \bar{S})$ on $G$, we define the mutuality tendency ratio cut function as follows.

$$
\operatorname{TRCut}(S, \bar{S})=\Theta_{\partial S}\left(\frac{1}{|S|}+\frac{1}{|\bar{S}|}\right),
$$

which represents the overall mutuality tendency across clusters balanced by the "sizes" of the clusters. Then, the clustering problem is formulated as a minimization problem with $K=2$ clusters. (More general cases with $|V| \geq K>2$ will be discussed in the next subsection.)

$$
\min _{S} \operatorname{TRCut}(S, \bar{S})
$$

Since $\Theta_{\partial S}=\Theta_{G}-\left(\Theta_{S}+\Theta_{\bar{S}}\right)$ holds true, we have

$$
\operatorname{TRCut}(S, \bar{S})=\left(\Theta_{G}-\left(\Theta_{S}+\Theta_{\bar{S}}\right)\right)\left(\frac{1}{|S|}+\frac{1}{|\bar{S}|}\right) .
$$

For a given graph $G$, the graph mutuality tendency $\Theta_{G}$ is a constant, the minimization problem in eq.116) is equivalent to the following maximization problem:

$$
\max _{S}\left\{\left(\Theta_{S}+\Theta_{\bar{S}}-\Theta_{G}\right)\left(\frac{1}{|S|}+\frac{1}{|\bar{S}|}\right)\right\}
$$

Hence, minimizing the cross-cluster mutuality tendency is equivalent to maximize the within-cluster mutuality tendency. Using the results presented in Theorem 11, we prove the following theorem which provides the solution to the above mutuality tendency optimization problem.

Theorem 2. Given the tendency Laplacian matrix $L_{T}=$ $D_{T}-T$, the signs of the eigenvector of $L_{T}$ corresponding to the smallest non-zero eigenvalue indicate the optimal solution $(S, \bar{S})$ to the optimization problem eq.(16).

Proof : Define the column vector $f_{S}=\left[f_{S}(1), \cdots, f_{S}(n)\right]^{T}$ with respect to a partition $S \cup \bar{S}=V$ as follows:

$$
f_{S}(i)=\left\{\begin{array}{ll}
\sqrt{|\bar{S}| /|S|} & \text { if } i \in S \\
-\sqrt{|S| /|\bar{S}|} & \text { if } i \in \bar{S}
\end{array} .\right.
$$

Then, by applying Theorem 1, we have

$$
\begin{aligned}
f_{S}^{T} L_{T} f_{S} & =\sum_{i \sim j} T_{i j}\left(f_{S}(i)-f_{S}(j)\right)^{2} \\
& =\left(\frac{|S|}{|\bar{S}|}+\frac{|\bar{S}|}{|S|}+2\right) \sum_{i \in S \sim j \in \bar{S}} T_{i j} \\
& =|V|\left(m_{\partial S}^{(\omega)}-m_{\partial S}^{(\mu)}\right)\left(\frac{1}{|\bar{S}|}+\frac{1}{|S|}\right), \\
& =|V| \Theta_{\partial S}\left(\frac{1}{|\bar{S}|}+\frac{1}{|S|}\right) .
\end{aligned}
$$

In addition, we have $f_{S}^{T} f_{S}=\left\|f_{S}\right\|^{2}=|V|$. Hence, Rayleighquoient for $L_{T}$ is

$$
\frac{f_{S}^{T} L_{T} f_{S}}{f_{S}^{T} f_{S}}=\operatorname{TRCut}(S, \bar{S}) \geq \lambda\left(L_{T}\right),
$$

where $\lambda\left(L_{T}\right)$ is the smallest non-zero eigenvalue of $L_{T}$. Here $\lambda\left(L_{T}\right)$ cannot be 0 , because we have the constraint
$f_{S} \perp \mathbf{1}$. From Theorem 11 1 is an eigenvector associated with eigenvalue 0 . Hence, the problem of minimizing eq.(16) can be equivalently rewritten as

$\min _{S} f_{S}^{T} L_{T} f_{S}$, s.t.: $f_{S} \perp \mathbf{1}$ in form of eq. (18), $\left\|f_{S}\right\|^{2}=|V|$.

Since the entries of the solution vector $f_{S}$ are only allowed to take values in form of eq.(18), this is a discrete optimization problem, which is known to be NP hard [25]. By relaxing the discreteness condition and allowing $f_{S}(i)$ to take arbitrary values in $\mathbb{R}$, we have the following relaxed optimization problem.

$$
\min _{f_{S} \in \mathbb{R}^{n}} f_{S}^{T} L_{T} f_{S}, \quad \text { s.t.: } f_{S} \perp \mathbf{1} \text {, and }\left\|f_{S}\right\|^{2}=|V| .
$$

The solution to this problem, i.e., the vector $f_{S}$, is the eigenvector corresponding to the smallest non-zero eigenvalue $\lambda\left(L_{T}\right)$. Hence, we can approximate the minimizer of $T R C u t(S, \bar{S})$ using the eigenvector corresponding to $\lambda\left(L_{T}\right)$. To obtain a partition of the graph, we need to convert the real-valued solution vector $f_{S}$ of the relaxed problem to an indicator vector. One way to do this [25] is to use the signs of $f_{S}$ as indicator function, where node $v_{i} \in S$, if $f_{S}(i) \geq 0$, and $v_{i} \in \bar{S}$, otherwise.

\section{B. Mutuality-tendency-aware spectral clustering: $K>2$}

For the case of finding $K>2$ clusters $S_{1} \cup \cdots \cup S_{K}=V$, we define the indicator vectors $h_{k}=\left(h_{1 k}, \cdots, h_{n k}\right)$,

$$
h_{i k}= \begin{cases}\frac{1}{\sqrt{\left|S_{k}\right|}} & \text { if } v_{i} \in S_{k} \\ 0 & \text { otherwise }\end{cases}
$$

where $i=1, \cdots, n$ and $k=1, \cdots, K$. Let $H$ denote the indicator matrix containing those $K$ indicator vectors as columns. Observe that $H^{T} H=I, h_{k}^{T} h_{k}=1$, and

$$
h_{k}^{T} L_{T} h_{k}=\frac{\Theta_{\partial S_{k}}}{\left|S_{k}\right|} .
$$

Define the mutuality tendency ratio cut $\operatorname{TRCut}\left(S_{1}, \cdots, S_{K}\right)$ for $K>2$ clusters as follows:

$$
\operatorname{TRCut}\left(S_{1}, \cdots, S_{K}\right)=\sum_{k=1}^{K} \frac{\Theta_{\partial S_{k}}}{\left|S_{k}\right|},
$$

where the ratio cut reduces to eq. (15) when $K=2$. The problem of minimizing TRCut can be formulated as

$$
\begin{aligned}
& \min _{S_{1}, \cdots, S_{K}} \operatorname{TRCut}\left(S_{1}, \cdots, S_{K}\right)=\min _{S_{1}, \cdots, S_{K}} \operatorname{tr}\left(H^{T} L_{T} H\right) \\
& \text { s.t.: } H^{T} H=I \text {, where } H \text { is defined in eq. 21. }
\end{aligned}
$$

One way of solving this problem is utilizing the method used in [25] by relaxing the discreteness condition to have a standard trace minimization problem as

$$
\min _{H \in \mathbb{R}^{|V| \times K}} \operatorname{tr}\left(H^{T} L_{T} H\right), \quad \text { s.t.: } H^{T} H=I
$$

The optimal solution $H$ contains the first $K$ eigenvectors of $L_{T}$ as columns. The clusters can be then obtained by applying the K-means algorithm on those $K$ eigenvectors. 
The solution obtained minimizes the mutuality tendency across clusters (which is equivalent to maximizing the within-cluster mutuality tendency).

Choice of $K$. We choose $K$, i.e., the total number of clusters, using the eigengap heuristic [25]. Theorem 1 shows that $L_{T}$ has all real eigenvalues. Denote the eigenvalues of $L_{T}$ in an increasing order, i.e., $\lambda_{1} \leq \cdots \leq \lambda_{n}$, The index of the largest eigengap, namely, $K:=\operatorname{argmax}_{2<K<n}(g(K))$, where $g(K)=\lambda_{K}-\lambda_{K-1}, K=2, \cdots, n$, indicates how many clusters there are in the network.

\section{EVALUATIONS}

In this section, we evaluate the performance of the mutuality-tendency-aware spectral clustering method by comparing it with various symmetrization methods based digraph spectral clustering algorithms. We only present the comparison results for the adjacency matrix symmetrization method, with objective matrix as $\bar{A}=\left(A+A^{T}\right) / 2$. For other settings, we obtained similar results and omit them here. We will 1) first test the performances using synthetic datasets, and then 2) apply our method to real online network datasets, e.g., Slashdot social network, and discover stable clusters with respect to mutual and one-way connections.

\section{A. Synthetic datasets}

We first consider synthetic datasets designed specifically to test the performance of our mutuality-tendency-aware spectral clustering method. We randomly generate a network, with 1000 nodes. There are 38000 directed edges (around 3.8\% of all directed node pair 1 ) in total, among which one third of them around 12666 edges are bidirectional, and two third of them around 25334 edges are unidirectional. Those nodes fall into 2 clusters, with 600 and 400 nodes respectively, where around $93.5 \%$ of the bidirectional edges are randomly placed within clusters, and around $80.8 \%$ of the unidirectional edges are randomly placed across clusters.

We show in Fig. 2(a)(i)-Fig. 2(a)(iii) that the traditional spectral clustering algorithm with $\bar{A}=\left(A+A^{T}\right) / 2$ as the objective results in clusters with 180 and 820 nodes respectively, which does not reflect the underlying structure (See Fig. 2(a)(i)-Fig. 2(a)(iii), because it clusters nodes without considering the stability difference between mutual connections and one-way connections. On the other hand, our proposed mutuality-tendency-aware spectral clustering method can cluster the nodes into groups with exactly 600 and 400 nodes (See Fig. 2(b)(i)-Fig. 2(b)(iii)), which clearly group nodes with more mutual (stable) connections together and separate nodes connected via one-way connections.

Furthermore, given the cluster mutuality tendency $\Theta_{S}$, we denote the average mutuality tendency of $S$ as $\theta_{S}=\Theta_{S} / N_{S}$, with $N_{S}=|S|(|S|-1) / 2$ as the total number of dyads in $S$.

\footnotetext{
${ }^{1}$ As we observed from real social networks, e.g., Slashdot.com [23], an online commenting network dataset, which will be discussed in the next section, the sparsity of its "core" network is around $0.19 \%$. Here, we choose $3.8 \%$, that is 20 times large of the real network sparsity, just for the ease of visualization of the clustering structure.
}

TABLE I

AVE. MUTUALITY TENDENCY COMPARISON ON SYNTHETIC DATASET

\begin{tabular}{|l||c|c|c|c|}
\hline$\cdot$ & $\theta_{G}$ & $\theta_{S}$ & $\theta_{\bar{S}}$ & $\theta_{\partial S}$ \\
\hline Tendency aware clustering & 0.0112 & 0.0172 & 0.0314 & $8.25 \mathrm{e}-5$ \\
Traditional clustering & 0.0112 & 0.0115 & 0.0202 & 0.0096 \\
\hline
\end{tabular}

Similarly, we have the average mutuality tendency of $G, \bar{S}$, and $\partial S$ as $\theta_{G}=\Theta_{G} / N_{d}, \theta_{\bar{S}}=\Theta_{\bar{S}} / N_{\bar{S}}$, and $\theta_{\partial S}=\Theta_{\partial S} /(|S||\bar{S}|)$, respectively. Table \ shows the average mutuality tendencies of the cluster results obtained by two methods, where we can see that the mutuality-tendency-aware spectral clustering algorithm can group nodes together with higher within-cluster tendencies than that by traditional spectral clustering. On the other hand, the cross-cluster tendency obtained using our method is very close to 0 , which means that the dyads across the clusters establish the mutual connections without any tendency (or purely independently). In addition, we generated synthetic dataset with $K>2$ clusters, and similar results are obtained shown in Fig. 3 ,

\section{B. Real Social Networks}

In the second set of simulations, we applied our mutualitytendency-aware spectral clustering algorithm to several real social network datasets, e.g., Slashdot [23], Epinions [19], and email communication network [12] datasets, and compare with various symmetrization methods based digraph clustering algorithms, such as $A=\left(A+A^{T}\right) / 2, A A^{T}$ and $F_{\pi}=\Pi P$. Here we only show the comparison results with adjacency matrix symmetrization based digraph spectral clustering on Slashdot dataset. All other settings lead to similar results and we omit them here for brevity.

Slashdot is a technology-related news website founded in 1997. Users can submit stories and it allows other users to comment on them. In 2002, Slashdot introduced the Slashdot Zoo feature which allows users to tag each other as friends or foes. The network data we used is the Slashdot social relation network, where a directed edge from $i$ to $j$ indicates an interest from $i$ to $j$ 's stories (or topics). Hence, two people with mutual connections thus share some common interests, while one-way connections infer that one is interested in the other's posts, but the interests are not reciprocated back. The Slashdot social network data was collected and released by Leskovec [23] in November 2008.

TABLE II

STATISTICS OF SLASHDOT SOCIAL NETWORK DATASET

\begin{tabular}{|c|c|}
\hline Nodes & 77360 \\
\hline Edges & 828161 \\
\hline Unidirectional edges & 110199 \\
\hline Bidirectional edges & 717962 \\
\hline Nodes in largest SCC & 70355 \\
\hline Edges in largest SCC & 818310 \\
\hline Unidirectional edges in largest SCC & 100930 \\
\hline Bidirectional edges in largest SCC & 717380 \\
\hline Nodes in the "core" component & 10131 \\
\hline Edges in the "core" component & 197378 \\
\hline Unidirectional edges in the "core" component & 21404 \\
\hline Bidirectional edges in the "core" component & 175974 \\
\hline
\end{tabular}



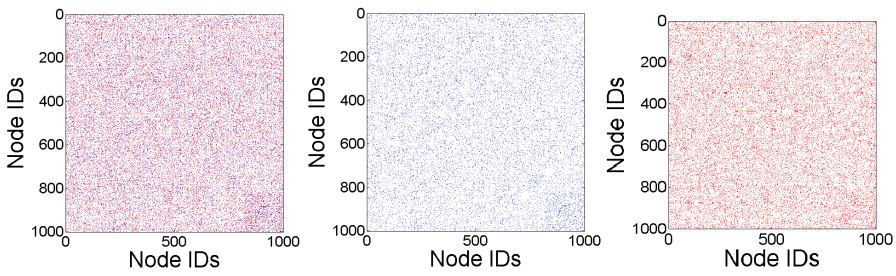

(a) (i)All edges, (ii)Bidirectional edges, (iii)Unidirectional edges
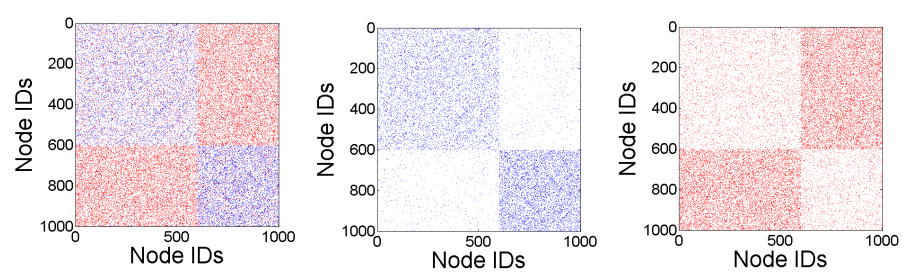

(b) (i)All edges, (ii)Bidirectional edges, (iii)Unidirectional edges

Fig. 2. Simulation results on synthetic dataset with $K=2$ clusters. Fig.2(a)(i)-Fig.2(a)(iii) show the clusters detected by traditional spectral clustering algorithm, and Fig.2(b)(i)-Fig.2(b)(iii) show the clusters extracted using our mutuality-tendency-aware spectral clustering algorithm.
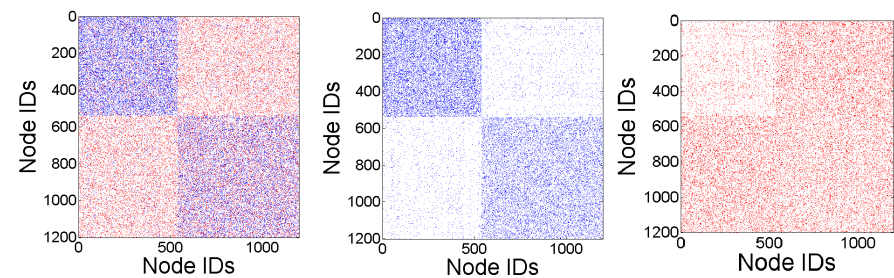

(a) (i)All edges, (ii)Bidirectional edges, (iii)Unidirectional edges
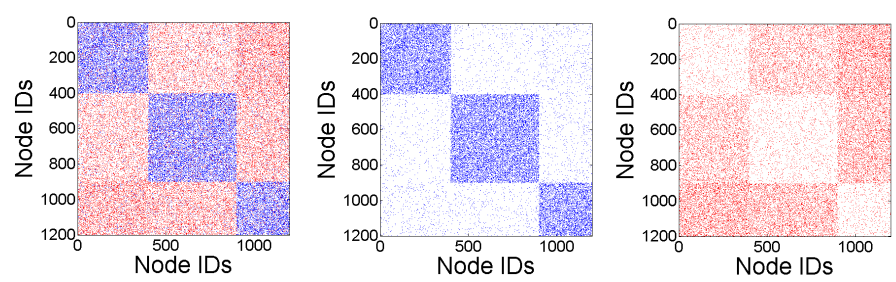

(b) (i)All edges, (ii)Bidirectional edges, (iii)Unidirectional edges

Fig. 3. This synthetic dataset is generated in $K=3$ clusters, with 500, 400 and 300 nodes, respectively. There are 54675 directional edges, among which 27336 edges are bidirectional and 27339 edges are unidirectional. We are randomly placed $90.02 \%$ of the bidirectional edges in clusters, and $89.6 \%$ of the unidirectional edges across clusters. Fig.3(a)(i)-Fig.3(a)(iii) show that traditional spectral clustering algorithm detects clusters with 661,538 and 1 entities, respectively, while our method identify correct clusters (See Fig.3(b)(i)-Fig.3(b)(iii)).
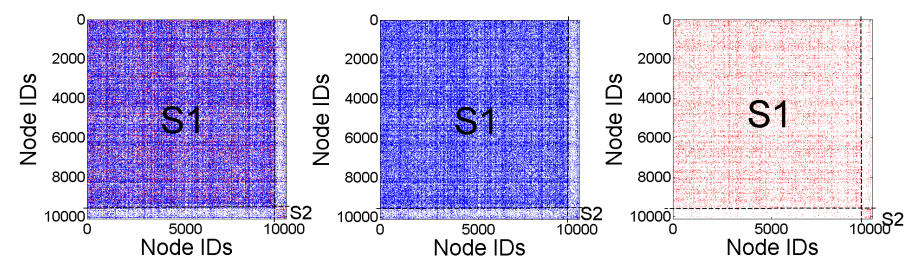

(a) (i)All edges, (ii)Bidirectional edges, (iii)Unidirectional edges
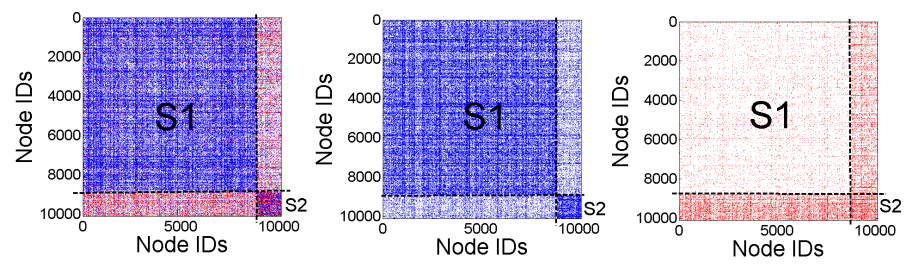

(b) (i)All edges, (ii)Bidirectional edges, (iii)Unidirectional edges

Fig. 4. Simulation results on Slashdot social network dataset. Fig.4(a)(i)-Fig.4(a)(iii) show the clusters detected by traditional spectral clustering algorithm, and Fig.4(b)(i)-Fig.4(b)(iii) show the clusters extracted using our mutuality-tendency-aware spectral clustering algorithm.

The statistic 2 are shown in Table It shows that the largest strongly connected component (SCC) include about 70355 nodes. Then, we remove those nodes with very low in-degrees and out-degrees, say no more than or equal to 2 . By finding the largest strongly connected component of the remaining graph, we extract a "core" of the network with 10131 nodes and 197378 edges, among which there are 21404 unidirectional edges and 175974 bidirectional edges, respectively.

In our evaluations, we observe that there is a large "core" of the network, and all other users are attached to this core network. In our study, we are interested in extracting the community structure from the "core" network.

When applying our spectral clustering algorithm to the "core" network, two clusters with 8892 and 1239 nodes are detected (shown in Fig 4(b) (i)-Fig 4(b) (iii)). In our result, a large portion (about $35.04 \%$ ) of cross-cluster edges are unidi-

\footnotetext{
${ }^{2} \mathrm{Here}$, the total number of edges is smaller than that is shown on the website [23], because we do not count for those selfloops.
}

rectional edges which in turn yield lower mutuality tendency across clusters. On the other hand, when using the traditional symmetrized $\bar{A}=\left(A+A^{T}\right) / 2$, two clusters with 9640 and 491 nodes are extracted instead (shown in Fig 4(a) (i)-Fig 4(a) (iii)). We can see that the clustering result obtained using the traditional spectral clustering method has only around $5.75 \%$ of the total edges across clusters as unidirectional edges, which boost up the mutuality tendency across clusters. However, in our clustering result, we have more unidirectional edges placed across clusters, which decreases the mutuality tendency across clusters. From Fig. 4(b)(i), we can clearly see that we have unidirectional (red) edges dominating the cross-cluster parts.

Table III shows the average mutuality tendency comparison between different clustering methods, where we can see that the mutuality-tendency-aware spectral clustering algorithm can group nodes together with higher within-cluster tendencies than that of traditional spectral clustering. 
TABLE III

AVE. MUTUALITY TENDENCY COMPARISON ON SLASHDOT DATASET

\begin{tabular}{|l||c|c|c|c|}
\hline. & $\theta_{G}$ & $\theta_{S 1}$ & $\theta_{S 2}$ & $\theta_{\partial S}$ \\
\hline Tendency aware clustering & 0.0017 & 0.0049 & 0.0028 & 0.00033 \\
Traditional clustering & 0.0017 & 0.0018 & 0.0021 & 0.00070 \\
\hline
\end{tabular}

\section{CONCLUSION}

In this paper, we establish a generalized mutuality tendency theory to capture the tendencies of clustered node pairs to establish mutual connections more frequently than those occur by chance. Based on our mutuality tendency theory, we develop a mutuality-tendency-aware spectral clustering algorithm that can detect stable clusters, by maximizing the withincluster mutuality tendency and minimizing the cross-cluster mutual tendency. Extensive simulation results on synthetic, and real online social network datasets, such as Slashdot, demonstrate that our proposed mutuality-tendency-aware spectral clustering method resolves more stable social community structures than traditional spectral clustering methods.

\section{REFERENCES}

[1] E. Berscheid and P. Regan. The psychology of interpersonal relationships. Pearson Prentice Hall, 2005.

[2] P. DeScioli, R. Kurzban, E. Koch, and D. Liben-Nowell. Best friends: Alliances, friend ranking, and the myspace social network. Perspectives on Psychological Science, 6(1):6-8, 2011.

[3] S. Golder, S. Yardi, and A. Marwick. A structural approach to contact recommendations in online social networks. Workshop on Search in Social Media (SSM), 2009.

[4] A. Gouldner. The norm of reciprocity: A preliminary statement. American sociological review, pages 161-178, 1960.

[5] L. Hagen and A. Kahng. New spectral methods for ratio cut partitioning and clustering. IEEE Transactions on Computer-Aided Design of Integrated Circuits and Systems, 11(9):1074-1085, 1992.

[6] F. Heider. Attitudes and cognitive organization. Journal of psychology, 21(1):107-112, 1946.

[7] M. Jamali, G. Haffari, and M. Ester. Modeling the temporal dynamics of social rating networks using bidirectional effects of social relations and rating patterns. In $W W W, 2011$.

[8] L. Katz and J. Powell. Measurement of the tendency toward reciprocation of choice. Sociometry, 18(4):403-409, 1955.

[9] M. Kurucz, A. Benczur, K. Csalogany, and L. Lukacs. Spectral clustering in telephone call graphs. In WebKDD 2007.

[10] H. Kwak, H. Chun, and S. Moon. Fragile online relationship: A first look at unfollow dynamics in twitter. In $C H I, 2011$.

[11] E. Leicht and M. Newman. Community structure in directed networks. Physical Review Letters, 100(11):118703, 2008.

[12] J. Leskovec, J. Kleinberg, and C. Faloutsos. Graph evolution: Densification and shrinking diameters. ACM Transactions on Knowledge Discovery from Data (TKDD), 1(1):2-es, 2007.

[13] J. Leskovec, K. Lang, and M. Mahoney. Empirical comparison of algorithms for network community detection. In $W W W, 2010$.

[14] Y. Li and Z.-L. Zhang. Random walks on digraphs, the generalized digraph laplacian and the degree of asymmetry. In LNCS WAW 2010, Stanford, CA, 2010. LNCS.

[15] U. Luxburg. A tutorial on spectral clustering. Statistics and Computing, 17:395-416, December 2007.

[16] H. Miller and D. Geller. Structural balance in dyads. Journal of Personality and Social Psychology, 21(2):135, 1972.

[17] N. Mishra, R. Schreiber, I. Stanton, and R. Tarjan. Clustering social networks. Algorithms and Models for the Web-Graph, 2007.

[18] K. Price, E. Harburg, and T. Newcomb. Psychological balance in situations of negative interpersonal attitudes. Journal of Personality and Social Psychology, 3(3):265, 1966.

[19] M. Richardson, R. Agrawal, and P. Domingos. Trust management for the semantic web. The SemanticWeb-ISWC, 2003.
[20] A. Roudrigues. Effects of balance, positivity, and agreement in triadic social relations. Journal of Personality and Social Psychology, 5(4):472, 1967.

[21] V. Satuluri and S. Parthasarathy. Symmetrizations for clustering directed graphs. In EDBT/ICDT, 2011.

[22] J. Shi and J. Malik. Normalized cuts and image segmentation. IEEE Trans. Pattern Anal. Mach. Intell., 22(8):888-905, 2000.

[23] Slashdot. dataset. http://snap.stanford.edu/data/soc-Slashdot0811.html.

[24] S. Smyth. A spectral clustering approach to finding communities in graphs. In SDM 2005.

[25] U. von Luxburg. A tutorial on spectral clustering. Technical Report No.TR-149, Max Planck Institute for Biological Cybernetics, 2006.

[26] X. Wang and I. Davidson. Flexible constrained spectral clustering. In KDD 2010, pages 563-572, 2010.

[27] J. Weston, C. S. Leslie, E. Ie, D. Zhou, A. Elisseeff, and W. S. Noble. Semi-supervised protein classification using cluster kernels. Bioinformatics, 21(15):3241-3247, 2005.

[28] A. Wolfe. Social network analysis: Methods and applications. American Ethnologist, 24(1):219-220, 1997.

[29] D. Zhou, J. Huang, and B. Schölkopf. Learning from labeled and unlabeled data on a directed graph. In ICML, 2005. 